\title{
Further Validity of the Short Version of the Activities-Specific Balance Confidence Scale in Patients with Multiple Sclerosis
}

\author{
(D) Zuhal Abasıyanık1,2, (D) Turhan Kahraman², (D) Pınar Yigit1, (D) Cavid Baba33, (D) Ozge Ertekin4, (D) Serkan Ozakbas3 \\ ${ }^{1}$ Dokuz Eylul University Graduate School of Health Sciences, Izmir, Turkey \\ 2lzmir Katip Celebi University Faculty of Health Sciences, Department of Physiotherapy and Rehabilitation, Izmir, Turkey \\ ${ }^{3}$ Dokuz Eylul University Faculty of Medicine, Department of Neurology, Izmir, Turkey \\ ${ }^{4}$ Dokuz Eylul University School of Physical Therapy and Rehabilitation, Izmir, Turkey
}

\section{Abstract}

Objective: There is scarce data on the utility of the short version of the Activities-specific Balance Confidence Scale in persons with multiple sclerosis (pwMS). Thus, this study aimed to expand the validity of the ABC-6 scale and compare it with the original version of the 16 -item ABC scale (ABC-16) in pwMS.

Materials and Methods: In total, 156 patients were included (median age: 35 years, $73.1 \%$ female and 26.9\% male) in this study. The ABC-16 and ABC-6, timed up-and-go test (TUG), TUG-cognitive, six-minute walk test, timed 25-foot walk, MS walking scale, single-leg stance test, modified fatigue impact scale (MFIS), brief international cognitive assessment for multiple sclerosis, Beck depression inventory-II (BDI-II), and epworth sleepiness scale (ESS) were assessed. Validity was assessed in terms of criterion, convergent, discriminant, and known-group validity.

Results: The correlation coefficient between the ABC- 6 and ABC-16 was $0.974(p<0.001)$. The ABC-16 and ABC-6 were strongly correlated with measures of the expanded disability status scale, all walking and balance tests, and physical and psychosocial subscores of MFIS ( $r s=-0.520$ to $-0.811, \mathrm{p}<0.05)$. Moderate correlations were found with a cognitive subscore of fatigue, cognitive processing speed, visuospatial memory, and BDI-II ( $r s=-0.321$ to $-0.446, p<0.05$ ). Low correlations were found in verbal memory and ESS scores ( $r s=-0.160$ to $-0.246, p<0.05)$. PwMS with a moderatesevere disability had significantly lower ABC-6 scores than that of patients with mild disability $(p<0.001)$.

Conclusion: The ABC- 6 demonstrated high validity for measuring balance confidence in pwMS. Our findings strengthen the clinical utility of the ABC-6 in pwMS.

Keywords: Multiple sclerosis, balance confidence, balance impairment, validity

\section{Introduction}

Multiple sclerosis (MS) is a neurodegenerative disease characterized by reduced motor and cognitive functions and fall risk $(1,2)$. A total of $56 \%$ of patients with MS (pwMS) fall in the previous three months, and $37 \%$ of those are recurrent (3). Balance confidence is deemed as a psychological aspect of balance-demanding activities and falls (4). Furthermore, a lower level of balance confidence is demonstrated as related to lower physical activity and participation $(5,6)$.

The 16-item Activities-specific Balance Confidence (ABC16) scale has been a widely used patient-reported outcome measure for pwMS and other populations to assess balance confidence (7). In addition, the ABC-16 was shown to be the best determinant of falls in pwMS and older adults (8-10). Hence, its use to evaluate or predict falls is recommended. However, proper fulfillment of the original scale requires approximately 5-20 min. Therefore, a six-item version of the ABC-16 scale, named the $A B C-6$, was created to save time and allow a rapid quantitative assessment in busy clinical settings (11). However, limited data is available about the validity of the ABC- 6 in pwMS. Therefore, further research is warranted to confirm its utilization in this population.

Wood et al. (12) reported moderate convergent validity and good internal consistency of the ABC-6 in pwMS. They also

Address for Correspondence: Zuhal Abasıyanık, Dokuz Eylul University Graduate School of Health Sciences, Izmir, Turkey E-mail: zuhalabasiyanik@gmail.com ORCID-ID: orcid.org/0000-0003-3086-8102

Received: 25.06.2021 Accepted: 02.08.2021

${ }^{\circ}$ Copyright 2021 by the Journal of Multiple Sclerosis Research published by Galenos Publishing House. 
revealed that both the ABC- 6 and $A B C-16$ had good sensitivity and specificity in distinguishing between pwMS with high fall risk and controls, whereas were less accurate at differentiating low and high fall risk of pwMS. Nevertheless, only physiologic profile assessment and fall history were investigated in their study. Results are promising for the use of $A B C-6$, but these findings should be confirmed considering the multifactorial nature of balance confidence. Therefore, the present study aimed to expand the validity of the ABC-6 in pwMS, investigating its correlations with walking, balance, fatigue, cognition, and psychosocial measures. A better understanding of related factors of balance confidence can guide rehabilitation strategies and ultimately help reduce activity limitation and encourage task performance.

\section{Materials and Methods}

\section{Participants and Procedure}

Data collected from the initial assessments of the longitudinal study registered to ClinicalTrials.gov (NCT03878836) were analyzed for this study. The study protocol was approved by the Ethics Board of Dokuz Eylul University (approval number: 2016/27-08). Data were extracted from a patient registry electronic system (iMed, Version 6.1; Merck Serono SA, Geneva, Switzerland).

The study included data from 156 pwMS. Eligible participants were selected according to the following inclusion criteria: a definite diagnosis of MS, Expanded Disability Status Scale (EDSS) below 7, and aged 18-65 years. Exclusion criteria included relapse within 30 days, neurological disease diagnosis other than MS, and severe cognitive impairment hindering assessment understanding. Written informed consent was taken from all participants.

\section{Outcome Measures}

Basic demographics, including age and sex, were recorded. The clinical information of participants was obtained from the current records. Neurological disability level was measured using the EDSS (13).

\section{Balance Confidence}

The primary outcomes were the ABC-16 and ABC- 6 . The ABC-16 scale includes 16 items related to daily life activities. All items are ranked from 0 (no confidence) to 100 (complete confidence), and the score of the questionnaire is calculated by dividing the total score obtained from 16 activities by $16(6,7,14)$. The ABC- 6 constitutes the six most challenging tasks (questions 5, 6, 13, 14,15 , and 16 ) of the $A B C-16$ and approximately takes 5 min. Therefore, the score of ABC- 6 is calculated by dividing the sum of the six items by six (11).

\section{Walking}

The Six-Minute Walk Test (6MWT) was implemented to assess the walking capacity. Participants were instructed to walk safely at their fastest speed in 6 min, according to a study by Motl et al. (15) The total distance covered during the 6 min was recorded in meters.

Timed-25 Foot Walking (T25FW) was carried out in a 7.62-meter long corridor; participants were asked to walk as fast and safely as possible to measure the walking speed. The average of two trials was recorded as the test score (16).

Timed Up-and-Go (TUG) test is a widely used instrument that assesses dynamic stability, mobility, turning, and rising out of a chair (17). The dual-task assessment was performed using the TUG test as a motor task and by adding a cognitive task, which is a serial 3 subtraction task. Serial subtraction tasks have been previously used to quantify dual-task cost in pwMS $(18,19)$.

The severity of perceived walking difficulties was assessed using the 12-item MS Walking Scale (MSWS-12). Higher scores indicate an increased impact of MS on walking (20).

\section{Balance}

The static standing balance was assessed using the Single-Leg Stance Test. Participants were asked to stand on their dominant feet with eyes open for $60 \mathrm{~s}$. The test ended when participants achieved $60 \mathrm{~s}$ or their feet touched the ground or their opposite extremity (21).

\section{Fatigue}

The Modified Fatigue Impact Scale (MFIS) is a patient-rated instrument that obtains information about the effects of fatigue and consists of 21 items. It involves physical, psychosocial, and cognitive subscales. Higher scores show more perceived fatigue $(22,23)$

\section{Cognition}

Brief International Cognitive Assessment for MS (BICAMS) composing the oral version of symbol digit modalities test (SDMT), brief visuospatial memory test-revised, and california verbal learning test (CVLT-II) were used in our study. The Turkish version of BICAMS was found valid and suitable for pwMS (24).

\section{Depression}

The severity of depressive symptoms was evaluated using the Beck Depression Inventory-II (BDI-II). Higher scores of BDI-II show enhanced depression symptom severity (25).

\section{Sleep}

The Epworth Sleepiness Scale (ESS) was used to evaluate daytime sleepiness. Higher scores of ESS indicate increased daytime sleepiness. The reliability of the Turkish form of ESS was found high (26).

\section{Statistics Analysis}

Data analysis was conducted using the IBM SPSS Statistics for Windows (Version 25.0. Armonk, NY: IBM Corp.). Data distribution was checked using the Kolmogorov-Smirnov test 
and investigation of the histogram and plots. Nonparametric tests were used as the ABC- 6 and ABC-16 did not show normal distribution. Spearman rank-order correlation was calculated to identify associations between the ABC -6 scale, ABC-16 scale, and other measures. Correlation coefficients of 0.1-0.29 were interpreted as small, 0.3-0.49 as moderate, and 0.5-1.0 as strong correlations. The validity was assessed in terms of criterion, convergent, discriminant, and known-groups validity by testing predefined hypotheses. The correlation coefficient between the $A B C-16$ and $A B C-6$ was explored to assess the criterion validity, and a strong correlation was expected. For examining the convergent validity, the following predefined hypotheses were constructed:

1. A strong correlation was expected between the $A B C-6$ and ABC-16 as they assess the same construct.

2. A moderate to strong correlation was expected between the ABC- 6 and walking and balance tests, fatigue, cognitive processing speed, and visuospatial memory as they measure the nonsimilar construct, but these factors may influence balance confidence based on previous research.

A low correlation was foreseen between the ABC- 6 and sleepiness and verbal memory for examining the discriminant validity as they do not measure balance-related constructs. Known-groups validity assesses whether an instrument can distinguish between known groups of patients. In this study, the ABC- 6 and ABC-16 scores were compared using the Mann-Whitney $U$ test between the disability groups. Groups were described according to the EDSS score as mild disability group (EDSS $\leq 3.5$ ) and moderatesevere (EDSS $>3.5$ ) (27). Significance was set at $p<0.05$.

\section{Results}

Demographic and clinical characteristics of 156 participants with pwMS are summarized in Table 1. The clinical course of most patients was relapsing-remitting MS (92.3\%).

The median score of the ABC-16 and ABC- 6 were 83.12 and 73.33, respectively. Table 2 displays the correlation coefficient between ABC-6, ABC-16, and other outcome measures. All the predetermined hypotheses for the validity assessment were confirmed. The correlation coefficient between $A B C$ 6 and $A B C-16$ was $0.974(p<0.001)$. The $A B C-6$ and $A B C-16$ were strongly correlated with measures of the EDSS, walking and balance tests, and physical and psychosocial subscores of fatigue. The strongest correlation between ABC-16, ABC6 , and other outcome measures was found among perceived walking difficulties assessed using the MSWS-12 ( $\mathrm{rs}=-0.811$, rs $=-0.768, p<0.001$; respectively). Moderate correlations were found with the cognitive subscore of fatigue, SDMT, BVMT-R, and depression. Low correlations were found in CVLT-II and ESS scores. Correlations coefficients between the ABC- 6 and ABC-16 and other measures were similar.

\section{Table 1. Demographic and clinical characteristics of participants}

Total $(\mathrm{n}=156)$

\begin{tabular}{|l|l|}
\hline Age (years) & $35(28.0-44.0)$ \\
\hline Gender, $\mathbf{n}(\%)$ & \\
\hline Female & $114(73.1 \%)$ \\
\hline Male & $42(26.9 \%)$ \\
\hline EDSS (0-10) & $1.5(0-2.0)$ \\
\hline Disease duration (years) & $2(2.0-11.37)$ \\
\hline Clinical course of MS, $\mathbf{n}(\%)$ & \\
\hline Relapsing-remitting & $144(92.3 \%)$ \\
\hline Secondary-progressive & $9(5.8 \%)$ \\
\hline Primary-progressive & $3(1.9 \%)$ \\
\hline
\end{tabular}

EDSS: Expanded disability status scale, MS: Multiple sclerosis

Table 3 shows that patients with higher EDSS reported lower scores in the ABC- 6 and $A B C-16$ scales, and differences were significant for both scales.

\section{Discussion}

The primary finding of our study revealed that the ABC6 test is strongly correlated with measures of neurological disability level, walking and balance tests, and fatigue in PwMS. Furthermore, an almost perfect correlation was found between them. These findings provide insights into the clinical utility of the ABC- 6 in pwMS by showing good validity, including criterion, convergent, discriminant, and known-groups validity.

The median ABC-16 score (83.12) was lower than that of the ABC- 6 score. This result indicates that ABC- 6 contains more challenging activities compared with $A B C-16$ for pwMS. The median EDSS score was 1.5 in our sample; however, the balance confidence level assessed using ABC-6 (73.33) was not high. This finding supports that selected activities (item 5: reach on tiptoes, item 6: standing on a chair to reach, item 13: being bumped into by people while walking through a mall, item 14: stepping off an escalator with rail, item 15: stepping off an escalator without holding onto the railing, and item 16: walking on an icy sidewalk) are challenging for pwMS.

Balance confidence refers to balance-related self-efficacy, which increases the possibility of the activity being accomplished regardless of physical $(7,28)$. The increasing amount of evidence indicates that balance confidence as measured using the ABC16 is a predictor of falls in pwMS (8-10). The ABC-16 has the highest explanatory value, whereas performance-based tests could not adequately explain falls in those without clinical disability (10). Few studies were reported; however, ABC-6 also has good sensitivity in distinguishing fallers or participants with high risk fall in Parkinson's disease (71.4\%) and pwMS (95\%), and values are similar to the $A B C-16(12,29)$. 


\begin{tabular}{|c|c|c|c|}
\hline & $\begin{array}{l}\text { Median (interquartile } \\
\text { range) }\end{array}$ & $\begin{array}{l}\text { Correlation } \\
\text { coefficient with } \\
\text { ABC-16 }\end{array}$ & $\begin{array}{l}\text { Correlation } \\
\text { coefficient with } \\
\text { ABC-6 }\end{array}$ \\
\hline \multicolumn{4}{|l|}{ Criterion validity } \\
\hline$A B C-16(0-100)$ & $83.12(58.43-95.56)$ & - & 0.974 \\
\hline ABC-6 (0-100) & 73.33 (45.83-95.0) & 0.974 & - \\
\hline EDSS (0-10) & $1.5(0-2.0)$ & -0.668 & -0.635 \\
\hline 6MWT (m) & $465(380-520)$ & 0.606 & 0.607 \\
\hline T25FW (s) & $4.75(4.34-5.65)$ & -0.614 & -0.632 \\
\hline TUG (s) & $6.78(6.15-8.41)$ & -0.630 & -0.635 \\
\hline MFIS-total (0-84) & $27.0(10.0-48.0)$ & -0.604 & -0.582 \\
\hline MFIS-physical (0-36) & $13.0(4.0-21.0)$ & -0.679 & -0.663 \\
\hline MFIS-cognitive (0-40) & $13.0(4.0-22.0)$ & -0.446 & -0.421 \\
\hline MFIS-psychosocial (0-8) & $3.0(0-5.0)$ & -0.543 & -0.524 \\
\hline SDMT & $49.0(41.0-56.0)$ & 0.321 & 0.335 \\
\hline BVMT-R & $28.0(22.0-31.0)$ & 0.334 & 0.329 \\
\hline BDI-II (0-63) & $9.0(5.0-15.0)$ & -0.423 & 0.384 \\
\hline \multicolumn{4}{|l|}{ Discriminant validity } \\
\hline CVLT-II & $53.0(42.0-61.0)$ & 0.224 & 0.246 \\
\hline
\end{tabular}

$\mathrm{p}<0.05$ for all correlation coefficients

ABC-16: 16-item activities-specific balance confidence, ABC-6: 6-item activities-specific balance confidence, EDSS: Expanded disability status scale, 6MWT: Six-minute walk test, T25FW: Timed 25-foot walk, TUG: Timed up-and-go, MSWS-12: Multiple sclerosis walking scale, SLST: Single-leg stance test, MFIS: Modified fatigue impact scale, SDMT: Symbol digit modalities Test: BVMT-R: Brief Visuospatial memory test-revised, BDI-II: Beck depression Inventory, CVLT-II: California verbal learning test, ESS: Epworth sleepiness scale

Table 3. Comparison of ABC-6 and ABC-16 scores between pwMS with different disability levels (known-groups validity)

\begin{tabular}{|l|l|l|l|}
\hline & Mild disability group $(n=133)$ & Moderate-severe disability group ( $n=23)$ & $p$ \\
\hline ABC-6 & $83.33(53.33-95.0)$ & $30.0(10.0-45.83)$ & $<0.001$ \\
\hline
\end{tabular}

$A B C$-16: 16-item activities-specific balance confidence, $A B C-6:$ 6-item activities-specific balance confidence

Wood et al. (12) revealed good reliability and moderate convergent validity of the ABC-6 in pwMS. The ABC-6 was negatively correlated with physiologic profile assessment and fall history. However, this study overlooked other factors thought to be associated with balance confidence. Our previous research showed that psychosocial factors are also related to balance confidence in a large sample of pwMS (30). Depression, fatigue, and cognitive processing speed assessed using SDMT were determinants of the ABC-16. The ABC- 6 was also correlated with these measurements in this present study. Our results suggest that the $A B C-6$ can reflect the relationship with these factors.
In accordance with previous studies on the $A B C-16$, the $A B C$ 6 was also correlated with physical measurements, including T25FW, 6MWT, TUG, TUG-cognitive, MSWS-12, and balance assessment $(6,31)$. The fact that the correlation values with physical tests are so close to the $A B C-16$ suggests that items included in the ABC-6 successfully reflect balance confidence. Therefore, the $A B C-6$ can also be used to save time in clinics or research.

\section{Study Limitations}

Most of the pwMS had mild disabilities, which could restrict the generalizability of findings. Notwithstanding this limitation, this study involves some strength, such as relatively large sample size and considerations of other dimensions that are yet to 
be investigated in validity studies involving the ABC- 6 scale in pwMS.

\section{Conclusion}

The ABC-6 showed good criterion, convergent, discriminant, and known-groups validity in pwMS. Our study expands the evidence promoting the utility of the ABC- 6 scale to assess balance confidence in pwMS. The ABC-16 and ABC-6 were associated with disability level, physical functions, cognition, fatigue, depression, and sleepiness in pwMS and showed similar correlations with these measures. Thus, the ABC- 6 can be used in research and clinical settings as it allows rapid and accurate assessment of balance confidence.

\section{Ethics}

Ethics Committee Approval:The Non-invasive Research Ethics Board of Dokuz Eylul University approved the study protocol (protocol number: 2959-GOA and approval number: 2016/2708).

Informed Consent: Written informed consent was taken from all participants.

\section{Authorship Contributions}

Surgical and Medical Practices: C.B., S.O., Concept: Z.A., T.K., C.B., O.E., S.O., Design: Z.A., PY., C.B., O.E., S.O., Data Collection or Processing: : Z.A., PY., Analysis or Interpretation: Z.A., T.K., Literature Search: Z.A., PY., C.B., O.E., S.O., Writing: Z.A., T.K., C.B., O.E., S.O.

Conflict of Interest: No conflict of interest was declared by the authors.

Financial Disclosure: The authors declared that this study received no financial support.

\section{References}

1. Compston A, Coles A. Multiple sclerosis. Lancet 2008;372:1502-1517.

2. Coote S, Comber L, Quinn G, Santoyo-Medina C, Kalron A, Gunn H. Falls in People with Multiple Sclerosis: Risk Identification, Intervention, and Future Directions. Int J MS Care 2020;22:247-255.

3. Nilsagård Y, Gunn H, Freeman J, Hoang P, Lord S, Mazumder R, Cameron M. Falls in people with MS--an individual data meta-analysis from studies from Australia, Sweden, United Kingdom and the United States. Mult Scler 2015;21:92-100.

4. Hadjistavropoulos T, Delbaere K, Fitzgerald TD. Reconceptualizing the role of fear of falling and balance confidence in fall risk. J Aging Health 2011;23:3-23.

5. Schmid AA, Van Puymbroeck M, Altenburger PA, Dierks TA, Miller KK, Damush TM, Williams LS. Balance and balance self-efficacy are associated with activity and participation after stroke: a cross-sectional study in people with chronic stroke. Arch Phys Med Rehabil 2012;93:1101-1107.

6. Nilsagård Y, Carling A, Forsberg A. Activities-specific balance confidence in people with multiple sclerosis. Mult Scler Int 2012;2012:613925. doi: 10.1155/2012/613927.

7. Powell LE, Myers AM. The Activities-specific Balance Confidence (ABC) Scale. J Gerontol A Biol Sci Med Sci 1995;50A:M28-M34. doi: 10.1093/ gerona/50a.1.m28.
8. Landers MR, Oscar S, Sasaoka J, Vaughn K. Balance confidence and fear of falling avoidance behavior are most predictive of falling in older adults: prospective analysis. Phys Ther 2016;96:433-442.

9. Tajali S, Shaterzadeh-Yazdi MJ, Negahban H, van Dieën JH, Mehravar M, Majdinasab N, Saki-Malehi A, Mofateh R. Predicting falls among patients with multiple sclerosis: comparison of patient-reported outcomes and performance-based measures of lower extremity functions. Mult Scler Relat Disord 2017;17:69-74.

10. Abasıyanık Z, Kahraman T, Ertekin Ö, Baba C, Özakbaş S. Prevalence and determinants of falls in persons with multiple sclerosis without a clinical disability. Mult Scler Relat Disord 2021;49:102771. doi: 10.1016/j. msard.2021.102771.

11. Peretz C, Herman T, Hausdorff JM, Giladi N. Assessing fear of falling: can a short version of the activities-specific Balance Confidence scale be useful? Mov Disord 2006;21:2101-2105.

12. Wood TA, Wajda DA, Sosnoff JJ. Use of a Short version of the activitiesspecific balance confidence scale in multiple Sclerosis. Int J MS Care 2019;21:15-21.

13. Kurtzke JF. Rating neurologic impairment in multiple sclerosis: an expanded disability status scale (EDSS). Neurology 1983;33:1444-1452.

14. Ayhan Ç, Büyükturan Ö, Kirdi N, Yakut Y, Güler Ç. The Turkish version of the activities specific balance confidence (ABC) scale: Its cultural adaptation, validation and reliability in older adults. Turk Geriatri Dergisi 2014;17:157-163.

15. Motl RW, Suh Y, Balantrapu S, Sandroff BM, Sosnoff JJ, Pula J, Goldman MD, Fernhall B. Evidence for the different physiological significance of the 6- and 2-minute walk tests in multiple sclerosis. BMC Neurol 2012;12:6.

16. Fischer JS, Rudick RA, Cutter GR, Reingold SC. The Multiple Sclerosis Functional Composite Measure (MSFC): an integrated approach to MS clinical outcome assessment. National MS Society Clinical Outcomes Assessment Task Force. Mult Scler 1999:5:244-250.

17. Sebastião E, Sandroff BM, Learmonth YC, Motl RW. Validity of the timed up and go test as a measure of functional mobility in persons with multiple sclerosis. Arch Phys Med Rehabil 2016;97:1072-1077.

18. Veldkamp R, Romberg A, Hämäläinen P, Giffroy $X$, Moumdjian L, Leone C, Feys P, Baert I. Test-retest reliability of cognitive-motor interference assessments in walking with various task complexities in persons with multiple sclerosis. Neurorehabil Neural Repair 2019;33:623-634.

19. Postigo-Alonso B, Galvao-Carmona A, Benítez I, Conde-Gavilán C, Jover A, Molina S, Peña-Toledo MA, Agüera E. Cognitive-motor interference during gait in patients with Multiple Sclerosis: a mixed methods Systematic Review. Neurosci Biobehav Rev 2018;94:126-148.

20. Dib H, Tamam Y, Terzi M, Hobart J. Testing patient-reported outcome measurement equivalence in multinational clinical trials: an exemplar using the 12-item Multiple Sclerosis Walking Scale. Mult Scler J Exp Transl Clin 2017:3:2055217317728740. doi: 10.1177/2055217317728740

21. Chrintz H, Falster O, Roed J. Single-leg postural equlllbnum test. Scand J Med Sci Sports 1991;1:244-246.

22. Armutlu K, Keser I, Korkmaz N, AkbiyikDI, SümbüloğluV, Güney Z, Karabudak R. Psychometric study of Turkish version of Fatigue Impact Scale in multiple sclerosis patients. J Neurol Sci 2007;255:64-68.

23. Learmonth YC, Dlugonski D, Pilutti LA, Sandroff BM, Klaren R, Motl RW. Psychometric properties of the Fatigue Severity Scale and the Modified Fatigue Impact Scale. J Neurol Sci 2013;331:102-107.

24. Ozakbas S, Yigit P, Cinar BP, Limoncu H, Kahraman T, Kösehasanoğulları G. The Turkish validation of the Brief International Cognitive Assessment for Multiple Sclerosis (BICAMS) battery. BMC Neurol 2017;17:208.

25. Sacco R, Santangelo G, Stamenova S, Bisecco A, Bonavita S, Lavorgna L, Trojano L, D'Ambrosio A, Tedeschi G, Gallo A. Psychometric properties and validity of Beck Depression Inventory II in multiple sclerosis. Eur J Neurol 2016;23:744-50.

26. Izci B, Ardic S, Firat H, Sahin A, Altinors M, Karacan I. Reliability and validity studies of the Turkish version of the Epworth Sleepiness Scale. Sleep Breath 2008;12:161-168. 
27. Motl RW, Learmonth YC. Neurological disability and its association with walking impairment in multiple sclerosis: brief review. Neurodegener Dis Manag 2014;4:491-500.

28. Fortinsky RH, Panzer V, Wakefield D, Into F. Alignment between balance confidence and fall risk in later life: Has over-confidence been overlooked? Health Risk Soc 2009;11:341-352.

29. Cole MH, Rippey J, Naughton GA, Silburn PA. Use of a short-form balance confidence scale to predict future recurrent falls in people with parkinson disease. Arch Phys Med Rehabil 2016;97:152-156.
30. Abasıyanık Z, Özdoğar AT, Sağıcı Ö, Kahraman T, Baba C, Ertekin Ö, Özakbaş S. Explanatory factors of balance confidence in persons with multiple sclerosis: Beyond the physical functions. Mult Scler Relat Disord 2020;43:102239. doi: 10.1016/j.msard.2020.102239.

31. Nogueira LA, Dos Santos LT, Sabino PG, Alvarenga RM, Santos Thuler LC. Factors for lower walking speed in persons with multiple sclerosis. Mult Scler Int 2013;2013:875648. doi: 10.1155/2013/875648. 